\title{
Correlation between tongue pressure and electrical activity of the suprahyoid muscles
}

\author{
Vanessa Santiago dos Reis ${ }^{(1)}$ \\ Taynara Gomes de Araújo(1) \\ Renata Maria Moreira Moraes Furlan ${ }^{(1)}$ \\ Andréa Rodrigues Motta ${ }^{(1)}$
}

(1) Universidade Federal de Minas Gerais, Belo Horizonte, Minas Gerais, Brazil. Research developed at Departamento de Fonoaudiologia, Universidade Federal de Minas Gerais, Belo Horizonte, Minas Gerais, Brasil.

Conflict of interest: Nonexistent

Received on: May 27, 2017

Accepted on: October 17, 2017

Mailing address:

Andréa Rodrigues Motta

Faculdade de Medicina da UFMG - Av. Professor Alfredo Balena, 190 sala 251

Santa Efigênia - Belo Horizonte, Minas Gerais, Brasil

CEP: $30130-100$

E-mail: andreamotta@ufmg.br

\section{ABSTRACT}

Objective: to investigate the correlation between the tongue pressure and the electrical activity of the suprahyoid muscles.

Methods: a across-sectional, observational and analytical study conducted with 15 men and 22 women. Each participant underwent simultaneous assessment of maximal tongue pressure through the lowa Oral Performance Instrument (IOPI) and the surface electromyography of the suprahyoid muscles. They were asked to press the tongue against the hard palate in the anterior and posterior region, with and without IOPI. The adopted significance level of the performed analyses was $5 \%$.

Results: there was a moderate and significant correlation only between suprahyoid electrical activity and tongue pressure in the posterior region. It was verified that the measured electrical potentials, when using the IOPI, were greater in the tasks of anterior pressure than in the tasks of the posterior one, bilaterally. Without using the IOPI, the electrical potentials were greater in the posterior pressure than in the anterior one, bilaterally. Finally, the values of lingual pressure were compared with the bulb positioned in the anterior and posterior parts, and the anterior tongue pressure was higher.

Conclusion: there was a moderate correlation between tongue pressure and electrical potential of the suprahyoid muscles, researched by the surface electromyography, only when performing activities with the posterior portion of the tongue.

Keywords: Tongue; Muscle Tone; Electromyography; Speech, Language and Hearing Sciences 


\section{INTRODUCTION}

The tongue participates in several functions of the stomatognathic system, such as chewing, swallowing and phonoarticulation ${ }^{1}$. Changes in tongue tonus may interfere with the orofacial myofunctional performance and impair the individual's quality of life ${ }^{2,3}$. The tongue tonus, when altered, can also influence dental positioning, since the dental arch is submitted to different strengths, in different amplitudes and by varied organs, such as cheeks, lips and tongue ${ }^{4}$. When one of these strengths excels it is possible that tooth movement occurs, especially if this strength is constantly exerted on the teeth 4 .

The evaluation of the tongue tonus then becomes of great relevance, allowing to define the intervention and monitoring when necessary ${ }^{5}$. However, this evaluation is usually performed in a perceptive way, since the means available to measure this parameter are scarce. This personal evaluation, based on the professional's experience, can generate divergence of opinion, especially when the examiners present little clinical practice $^{6}$ Thus, the development and application of objective methods have been expanded in Orofacial Myology 5 .

The lowa Oral Performance Instrument (IOPI) is a device that provides numerical data about tongue pressure and resistance. It consists of an air bulb connected to a pressure transducer and has been increasingly used in several countries in research and clinical practice ${ }^{7}$ Researchers have used IOPI to measure the tongue of adults ${ }^{8}$, children and adolescents $^{9}$, individuals with dysphagia ${ }^{10}$, cleft lip and palate $^{11}$, obstructive sleep apnea ${ }^{12}$, head and neck cancer $^{13}$, muscular dystrophy ${ }^{14}$, Parkinson's disease ${ }^{15}$, individuals who suffered head injury ${ }^{16}$ and other changes. Besides, data provided by the IOPI were used to prove the efficacy of Speech-Language Pathologyto increasethe tongue strength ${ }^{17,18}$. And the exercise of pressing the IOPI bulb against the palate proved to be efficient in improving the swallowing of individuals who suffered cerebrovascular accident ${ }^{19}$. Moreover, researches carried out with adults $^{7}$ and elderly ${ }^{20}$ showed that the IOPI tongue pressure values presented acceptable reliability.

Another method used in the indirect evaluation of tongue strength is the surface electromyography (EMGs) of the suprahyoid muscles. The examination captures the potentials of action generated in muscular contractions and allows the comparison of these values in relation to the amplitude and duration of the movement ${ }^{21-25}$.

The suprahyoid muscles play an important role during swallowing because they are involved in the laryngeal elevation ${ }^{26}$. One study found that the increased tongue pressure against the palate coincided with increased suprahyoid muscle activity ${ }^{26}$, suggesting that tongue pressure exercises on the palate are indicated to strengthen not only the intrinsic musculature of the tongue, but also of the supra-hyoid muscles, improving airway protection during swallowing in patients with dysphagia. Thus, some studies use surface EMG of the suprahyoid musculature to compare tongue strength training exercises used in the clinical practice of dysphagia ${ }^{21,22,24}$.

No studies were found comparing the electrical activity of the suprahyoid muscles during tongue pressure activities separately performed in the anterior and posterior palate region. This analysis will allow to suggest which of these positions is best indicated as an exercise for the rehabilitation of the suprahyoid muscles.

Thus, the present research aims to analyze if there is a correlation between the values found in the tongue pressure measurements obtained through the lowa Oral Performance Instrument (IOPI) with the bulb in anterior and posterior position and the electrical potential of the suprahyoid muscles researched by surface electromyography.

\section{METHODS}

This is a cross-sectional, observational and analytical study, carried out at the Observatório de SaúdeFuncional em Fonoaudiologia (Functional Health Observatory in Speech-Language Pathology) of the Department of Speech-Language Pathology, Universidade Federal de Minas Gerais, Brazil, with a non-probabilistic sample. Thirty seven individuals participated in the study, 15 men and 22women with a mean age of 24 years. The work was approved by the Research Ethics Committee of the institution of origin under number 0515.0.203.000-11.

Inclusion criteria were: signature of the free and informed consent, age between 18 and 50 years, absence of cognitive or structural changes in the orofacial and cervical region, neurogenic diseases, oral lesions that caused pain or discomfort and lack of suction movement of tongue on the palate. Inclusion criteria also included: do not use drugs that lead to muscle weakness and report allergy to the materials 
used. These data were collected through interviews. Exclusion criteria were: do not tolerate the IOPI bulb in the oral cavity and do not perform all requested tongue strength measurements. Data from EMGs that presented excessive noise, making it impossible to analyze them, were excluded as recommended by Leniuset al. ${ }^{23}$.

The study was spread by the researchers through invitations in informal conversations with students and staff of the institution, as well as with acquaintances of the researchers. The people with interest to participate attended the evaluation place at the pre-established time and date. At the meeting, participants were first explained about the research, the risks and benefits generated, followed by the presentation of the free and informed consent and the signature of it.

All participants were submitted to medical history analysis (for verification of the inclusion criteria) and clinical evaluation, based on the MBGR ${ }^{27}$ protocol, which included the items: tongue aspects, lingual frenulum and occlusion. Besides, an item was added about the floor of the mouth, which, according to the subjective evaluation, was classified as elevated or without elevation. The clinical evaluation was conducted blindly by two evaluators. Through this assessment the researchers evaluated the existence of some change that could interfere in the accomplishment of the activities proposed in the research.

In an acoustically treated room, each individual sat in a chair without a headrest, with the back resting, relaxed hands on the legs and feet resting on the floor on a rubber mat ${ }^{28}$. The individuals were informed about the characteristics of the equipment and trained for the proper execution of the movements. After, the participants were simultaneously evaluated through IOPI and surface electromyography.

\section{Evaluation with IOPI}

The IOPI consists of an air bulb $(3.5 \mathrm{~cm}$ long and $1 \mathrm{~cm}$ in diameter), a pressure transducer, a $1.5 \mathrm{~cm}$ plastic tube that connects the bulb to the transducer and a LCD screen. The IOPI bulb was positioned in two regions: first in the anterior region of the hard palate, just behind the alveolar papilla, and secondly in a more posterior region, parallel to the first lower molars ${ }^{7}$.

Positioning the instrument in the anterior region of the hard palate, the individual was asked to press it with the tongue toward the palate with the greatest possible strength for 2 seconds. This procedure was performed three times, with a one-minute rest interval between them. The bulb was then positioned in the posterior intraoral region and the tongue compression task on the hard palate was performed using the posterior region of the tongue in three series with the same duration, frequency and rest. The researchers provided verbal encouragement during the activities.

As the air bulb was pressed by the tongue, the device picked up the generated pressure change. The values were measured in $\mathrm{kPa}$ and were visualized on the LCD screen of the device itself.

\section{Evaluation with EMG}

Concomitantly with the IOPI evaluation, an electromyograph(EMG System do Brasil Ltda ${ }^{\circledR}$ ), in the eight-channel EMG800C-832 version, was used, coupled to a computer, using the manufacturer's Software (Aq Dados, version 5.05, Lynx Tecnologia Eletrônica LTDA) for data acquisition and processing. The equipment recorded the muscular electric activity in microvolts $(\mu \mathrm{V})$ and the signal was filtered through high pass filters of $20 \mathrm{~Hz}$ and low pass of $500 \mathrm{~Hz}$, amplified with gain of $1000 \mathrm{x}$ and common mode rejection ratio $>120 \mathrm{~dB}$. The data were processed by a 16-bit analog-digital converter (EMG System do Brasil Ltda ${ }^{\circledR}$ ) with a sampling frequency of $2 \mathrm{KHz}$. The active electrodes had an amplification gain of 20x. Of the eight present channels, only two were used, the others being disabled. Three electrodes were used: one reference (ground) and two active.

A gauze soaked in $70 \%$ alcohol solution was used at the electrode fixation sites to remove excess of oil from the skin, allowing better conduction of the actionpotentialsand reduction of the system impedance.

The reference electrode was placed on a prominent bone, opting for the lateral epicondyle of the humerus, according to the manufacturer's instructions. The reference electrodes used were $3 \mathrm{M}^{\circledR}$ brand, disposable, pre-gelled, rectangular and self-adhesive. The electrical signals were obtained by using pre-gelled, circular, double and self-adhesive $\mathrm{Hal}^{\circledR}$ brand disposable surface electrodes $(\mathrm{Ag} / \mathrm{AgCl})$, with $10 \mathrm{~mm}$ in diameter and $20 \mathrm{~mm}$ of inter-electrode distance center to center, bilaterally positioned on the skin in the submental region, between the mandible and the hyoid bone ${ }^{23}$. These captured the electrical potentials generated by the muscles during their action. For the positioning of these electrodes, the participant was asked to strongly press the hard palate with the tongue in order to locate the most prominent area of the suprahyoid region. The fixation followed the longitudinal direction of the muscle 
bundles bilaterally to minimize the possible interference of the adjacent musculature. Thus, during the tasks performed with the IOPI, the electrical potentials of the suprahyoid muscles were captured for 10 seconds in each activity, and the values found in RMS, of each individual, were analyzed.

Considering that the present study seeks to identify a possible correlation between IOPI and EMG, since the first is not accessible to the clinician in Brazil, the electrical potentials were also researched in the same tongue movements against the palate, without the bulb. For this, the same duration, frequency and rest time were used.

\section{Normalization}

Before initiating activities with the IOPI, the participant performed the normalization task ${ }^{29}$ through the exercise of suctioning the tongue against the palate with strong pressure. Each participant performed three suctions for 5 seconds, and the mean RMS of these values was recorded as the normalization value of the participant. There were intervals of 60 seconds between each suction.

\section{Data Analysis}

Sections in the collection of the electromyographic signals were selected in order to obtain the analyzed parameters. In each performed activity the double electrodes captured the electrical potentials of the suprahyoid muscle bundles on the right and left. Thus, each performed activity provided two collections. Each activity was captured in a window of 10 seconds and, in the normalization, the individual performed the tongue suction exercise with strength for 5 seconds. The participant performed the other activities for 2 seconds. Some collections were excluded due to excessive noise.

As two researchers (A and $B$ ) were responsible for defining the sections of the collections, for a greater reliability a third evaluator (C) replicated $20 \%$ of the sample. There was a very strong and significant positive correlation in the analyses (Table 1). For classification of the correlation coefficient, value lower than 0.3 indicates negligible correlation; value higher than or equal to 0.3 and lower than 0.5 indicates weak correlation; value equal to or higher than 0.5 and lower than 0.7 indicates moderate correlation; value equal to or higher than 0.7 and lower than 0.9 indicates strong correlation; and a value higher than or equal to 0.9 indicates a very strong correlation ${ }^{30}$.

Table 1. Correlation between the selected sections for analysis by the researchers

\begin{tabular}{lc}
\hline Variables & Spearman Coefficient of correlation \\
\hline Right side & \\
Normalization task & $0.90^{\star}$ \\
EMG - anterior pressure without IOPI & $1.00^{*}$ \\
EMG - posterior pressure without IOPI & $1.00^{*}$ \\
EMG - anterior pressure with IOPI & $1.00^{*}$ \\
EMG - posterior pressure with IOPI & $1.00^{\star}$ \\
\hline Left side & \\
Normalization task & $0.93^{*}$ \\
EMG - anterior pressure without IOPI & $1.00^{*}$ \\
EMG - posterior pressure without IOPI & $1.00^{*}$ \\
EMG - anterior pressure with IOPI & $0.95^{\star}$ \\
EMG - posterior pressure with IOPI & $0.95^{*}$ \\
\hline
\end{tabular}

* Significance level of $5 \%$ 
To define the sections to be analyzed, the three evaluators followed the following procedures. When the mean of the signal exceeded two standard deviations of the mean of the noise ${ }^{29}$, it was established that the individual initiated the muscle contractions from the performed activities. For normalization, the data of the first second of contraction were discarded and the next three seconds were analyzed, also discarding the end of the signal. In the other activities, the entire signal was used, considering as the end of the contraction the last point where the mean of the signal is above two standard deviations of the mean of the noise.

The variables analyzed in the study were: tongue pressure values in $\mathrm{kPa}$ obtained by IOPI in anterior and posterior position; values of muscular electric activity in $\mu \mathrm{V}$ obtained through the EMG of the suprahyoid muscles, with and without the use of IOPI, in anterior and in posterior positions, both on the right and on the left sides.
The data were initially analyzed through measures of central tendency and dispersion. To evaluate the correlation of the data the Spearman coefficient was used and, in the comparison of the samples, the Wilcoxon test. STATA software (Stata Corporation, College Station, Texas), version 12.0 was used, considering a $5 \%$ significance level.

\section{RESULTS}

The measures of central tendency and dispersion of the data collected in the analyzed sample can be observed in Table 2. The electric potential data (in $\mu \mathrm{V}$ ) captured from muscle bundles positioned on the left tended to present higher values than from the ones on the right. After normalized, the values without the IOPI bulb were higher on the right and, with the bulb, on the left. As the collection performed with the IOPI was unique, the data were only duplicated in the table.

Table 2. Data collected through the lowa Oral Performance Instrument and the surface electromyography, with and without normalization

\begin{tabular}{llllllll}
\hline Variables & N & Mean & SD & Median & IQR & Minimum & Maximum \\
\hline Right Side & & & & & & & \\
Normalization task $(\mu \mathrm{V})$ & 37 & 50.4 & 37.1 & 35.3 & 39.7 & 13.4 & 168.6 \\
$\quad$ EMG - anterior pressure without IOPI $(\mu \mathrm{V})$ & 36 & 36.9 & 25.6 & 31.1 & 15.4 & 15.8 & 150.7 \\
$\quad$ EMG - posterior pressure without IOPI $(\mu \mathrm{V})$ & 36 & 48.7 & 42.7 & 33.3 & 29.3 & 11.4 & 245.5 \\
EMG - anterior pressure with IOPI $(\mu \mathrm{V})$ & 37 & 47.2 & 32.2 & 39.1 & 25.8 & 21.1 & 180.1 \\
$\quad$ EMG - posterior pressure with IOPI $(\mu \mathrm{V})$ & 37 & 59.0 & 64.5 & 45.1 & 27.7 & 15.1 & 393.6 \\
Normalized EMG - anterior pressure without IOPI (\%) & 36 & 87.8 & 38.6 & 84.4 & 47.6 & 12.4 & 198.7 \\
$\quad$ Normalized EMG - posterior pressure without IOPI (\%) & 36 & 109.4 & 53.7 & 96.9 & 70.8 & 29.6 & 228.9 \\
Normalized EMG - anterior pressure with IOPI (\%) & 37 & 114.6 & 66.3 & 105.2 & 47.0 & 23.3 & 360.6 \\
Normalized EMG - posterior pressure with IOPI (\%) & 37 & 131.7 & 74.8 & 124.2 & 57.5 & 20.0 & 321.6 \\
$\quad$ IOPI - bulbin the anterior position $(\mathrm{kPa})$ & 37 & 48.7 & 12.4 & 45.8 & 19.3 & 25.7 & 78.7 \\
$\quad$ IOPI - bulbin the posterior position $(\mathrm{kPa})$ & 37 & 38.4 & 14.6 & 39.0 & 18.0 & 12.0 & 66.3 \\
\hline Left Side & & & & & & & \\
Normalization task $(\mu \mathrm{V})$ & 36 & 56.6 & 46.7 & 40.5 & 44.5 & 12.4 & 239.0 \\
EMG - anterior pressure without IOPI $(\mu \mathrm{V})$ & 35 & 40.3 & 29.8 & 29.8 & 20.4 & 15.4 & 167.1 \\
EMG - posterior pressure without IOPI $(\mu \mathrm{V})$ & 35 & 55.8 & 57.5 & 39.0 & 35.2 & 10.7 & 332.6 \\
EMG - anterior pressure with IOPI $(\mu \mathrm{V})$ & 36 & 53.8 & 38.6 & 45.8 & 28.8 & 19.0 & 214.6 \\
EMG - posterior pressure with IOPI $(\mu \mathrm{V})$ & 36 & 68.7 & 85.7 & 44.9 & 40.5 & 12.0 & 526.6 \\
Normalized EMG - anterior pressure without IOPI (\%) & 35 & 86.4 & 37.8 & 84.6 & 55.1 & 16.2 & 194.8 \\
Normalized EMG - posterior pressure without IOPI (\%) & 35 & 107.0 & 45.8 & 105.8 & 75.0 & 25.2 & 216.3 \\
Normalized EMG - anterior pressure with IOPI (\%) & 36 & 118.8 & 70.7 & 100.3 & 52.8 & 22.7 & 345.2 \\
Normalized EMG - posterior pressure with IOPI (\%) & 36 & 131.9 & 72.5 & 122.8 & 78.2 & 14.4 & 352.3 \\
IOPI - bulb in the anterior position $(\mathrm{kPa})$ & 37 & 48.7 & 12.4 & 45.8 & 19.3 & 25.7 & 78.7 \\
IOPI - bulb in the posterior position $(\mathrm{kPa})$ & 37 & 38.4 & 14.6 & 39.0 & 18.0 & 12.0 & 66.3 \\
\hline
\end{tabular}

Legend: N: number of participants; SD: Standard Deviation; IQR: Interquartile Range; EMG: electromyography; IOPI: lowa Oral Performance Instrument 
In the verification of the correlation between the IOPI and the non-normalized EMG data, a moderate and significant classification was found only between the findings of the posterior tongue activities on both sides (Table 3).

Considering that in the present study the EMG collections were performed with and without the IOPI, it was sought to verify if the presence of the instrument would interfere with the values.

In the normalized data (Table 4), when comparing the presence or absence of IOPI, it was verified that the electrical potentials measured with IOPI were higher only in the tasks of anterior pressure, both on the right and on the left, compared to the values obtained without the use of IOPI. Regarding the pressure site, a significant difference was observed only without the use of the IOPI: the posterior pressure generated greater electrical potentials than the anterior one both on the right and on the left. The found pattern of responses for the non-normalized data was the same as the one obtained with the normalized data.

Finally, the values obtained using the IOPI were compared when the bulb was positioned in the anterior part $(48.7 \mathrm{kPa})$ and in the posterior one $(38.4 \mathrm{kPa})$ of the palate. According to the Wilcoxon test $(p<0.001)$ the anterior tongue pressure was higher.

Table 3. Correlation between the findings of the lowa Oral Performance Instrument and non-normalized surface electromyography

\begin{tabular}{ccc}
\hline Tongue pressure $\mathbf{( k P a )}$ & EMG $(\mathbf{m V})$ \\
\hline & & 0.19 \\
IOPI - bulb in the anterior position & & $0.45^{\star}$ \\
IOPI - bulb in the posterior position & & \\
\hline & Left side & 0.27 \\
IOPI - bulb in the anterior position & & $0.50^{\star}$ \\
\hline $\mathrm{OPI}$ - bulb in the posterior position & & \\
\hline
\end{tabular}

* Spearman's Coefficient - significance level of $5 \%$

Table 4. Comparison of the findings of normalized surface electromyography with and without the use of the lowa Oral Performance Instrument

\begin{tabular}{|c|c|c|c|}
\hline & With the IOPI & Without the IOPI & p-value \\
\hline \multicolumn{4}{|c|}{ Right side } \\
\hline Anterior pressure (\%) & 114.6 & 87.8 & $0.008^{*}$ \\
\hline Posterior pressure (\%) & 131.7 & 109.4 & 0.066 \\
\hline$p$-value & 0.058 & $0.005^{*}$ & ---- \\
\hline \multicolumn{4}{|c|}{ Left Side } \\
\hline Anterior pressure (\%) & 118.8 & 86.4 & $0.003^{*}$ \\
\hline Posterior pressure (\%) & 131.9 & 106.9 & 0.098 \\
\hline $\mathrm{p}$-value & 0.068 & $0.007^{*}$ & ---- \\
\hline
\end{tabular}

* Wilcoxon Test - significance level of $5 \%$

\section{DISCUSSION}

The IOPI has emerged as an instrument that provides numerical data on the pressure and resistance of the tongue. But its use is restricted in researches in Brazil. This way, the access to an instrument that correlates with the tongue pressure becomes relevant. However, according to the analysis, the correlation is moderate when the bulb is posteriorly positioned and weak when in anterior position, suggesting that independent aspects are evaluated in the examination. This result confirms what was found by other authors: the EMGs of the suprahyoid muscles do not adequately represent the strength of the tongue in pressure activities of this organ against the palate ${ }^{23}$. These authors ${ }^{23}$ explain that the two tests capture information from different muscles. While the EMGs capture the activity of the mylohyoid, geniohyoid and anterior belly of the 
digastric muscles, with minimal contribution of the genioglossus one, the tongue pressure measurements on the palate are generated mainly by the genioglossus muscle, with a lower contribution of the suprahyoid ones $^{23}$.

Considering that the present study sought ways to infer about the tongue pressure since IOPI is not commercialized in Brazil, measures were taken without the IOPI, reproducing the same movements performed in the use of the instrument. It was verified that only the anterior measures are sensitive to the presence of the instrument in the oral cavity and, with the presence of the bulb in the anterior intraoral region, the electromyographic results were higher. This result suggests a greater participation of the suprahyoid muscles with the use of the IOPI, possibly for the support and compression of the object against the hard palate, leading to the capture of more electric potentials. Another study ${ }^{26}$ discussed the impact of bulb presence on the magnitude of the generated pressure. The authors suggest that the presence of the bulb alters the magnitude of the lingual pressure.

The concentration of intrinsic muscle tissue varies according to the tongue region, being higher in the posterior region $(57.3 \%)$ compared to the anterior $(25.9 \%)$ and medium $(44.4 \%)^{31}$ ones. It is believed that the suprahyoid electrical activity captured in the task of tongue lift against the palate differed between the posterior and anterior regions not only due to the difference in lingual muscle concentration, but also due to the presence of other muscles recruitment, such as the extrinsic ones of the tongue.The tongue pressure against the palate in the anterior region recruits more the genioglossus muscle than the tongue pressure against the palate in the posterior region, position in which the styloglossus and palatoglossal muscles are activated. Since EMGs are sensitive to muscle activation, tasks involving different muscles present different results ${ }^{23}$.

When comparing the electromyographic values according to the tongue pressure site (anterior or posterior region), it was observed that the posterior pressure generated greater electrical potentials than the anterior one, but only without the use of the IOPI bulb, suggesting that the IOPI also changes the pattern of muscle contraction, not just the magnitude of the strength.

It is worth highlighting that before the collection some participants requestedrepetitions of the posterior movements, in order to better understand the pressure that should be exerted on the hard palate with the posterior part of the tongue. These individuals only performed the activities after demonstrating an understanding of the proposed exercises, but this difficulty of perception and accomplishment of the posterior movement may have influenced the results, leading to contractions of different muscles during the posterior tongue exercises and greater electromyographic findings in these activities.

When comparing the values found only with the IOPI instrument, there was a greater tongue pressure in the anterior position, possibly suggesting a higher tonus at the apex of the tongue and not in the posterior region of it, despite the greater muscular concentration in the posterior region ${ }^{31}$. These findings, higher in anterior than in posterior regions, were also cited by another study $^{32}$, whose authors assessed lingual pressure during swallowing. The higher pressure values found in the anterior region of the tongue may be explained by the frequent strength that this region of the tongue performs against the palate when initiating the anteroposterior movement of propulsion of the bolus during swallowing ${ }^{32}$.

The predominant muscle fiber type in each region of the tongue also seems to contribute to greater strength of the anterior region. Muscle strength and fatigue resistance are determined by the characteristics of the muscle fibers, especially the diameter, and by their bio-energetic capacity to produce adenosine triphosphate (ATP), which provides energy for the contraction. Muscle fibers can be categorized in type I and type II. Type I fibers are slower in the contraction, but are more resistant to fatigue due to the greater capacity to produce ATP by aerobic metabolism. Moreover, they are relatively smaller in diameter than Type II fibers and therefore have less capacity of strength generation. The fast-twitch type II fibers are wider in diameter and therefore have a greater capacity ofstrength generation, but are less resistant to fatigue. They can be subdivided into types IIA, IIB, IIAB and IIC. There is also an additional type of fiber, type IM, which is present especially in human masticatory muscles. IIC and IM fibers have intermediate characteristics between types I and II. The type II fibers predominate in the anterior region, capable of generating great strength, but during a small period, because they quickly enter into fatigue. In the posterior region, there is a predominance of type I fibers, which generate less strength, but are more resistant to fatigue, besides the IM and IIC types, which, 
due to their intermediate characteristics, are adaptable to the several finely modulated oral actions ${ }^{33}$.

It isworth highlighting that the muscle fibers in the posterior region of the tongue are in horizontal orientation $^{31}$, while the anterior region presents perpendicular and parallel fibers. Gingrich and colleagues suggest that a smaller amount of fibers in the anterior region of the tongue, arranged perpendicularly to the bulb, may exert a greater strength than a greater amount of fibers in the posterior region of the tongue arranged horizontally to the bulb ${ }^{32}$.

Among the limitations found in this study, it was noticed difficulty in understanding and performing of the activities involving the posterior musculature of the tongue and the capture of electrical potentials of different muscles, already mentioned in previous articles ${ }^{23,29}$. Besides, as the bulb used in the research did not remain fixed in the intraoral region of the mouth, and as the coating material of this artifact is a plain plastic, some participants reported thatit moved with the pressure on the object, making it difficult to perform the tasks. Thus, this difficulty may have interfered in the results of both IOPI and electromyography, since these muscle contractions, originated from the attempt to paralyze the bulb, were possibly captured by the EMG.

\section{CONCLUSION}

The present study showed a moderate correlation between the values found in the tongue pressure measurements obtained by the lowa Oral Performance Instrument (IOPI) and the electrical potential of the suprahyoid muscles as researched by surface electromyography, only when performing activities with the posterior portion of the tongue.

\section{ACKNOWLEDGMENT}

The authors are grateful to Fundação de Amparo à Pesquisa de Minas Gerais - FAPEMIG for funding the project (Process CDS - APQ-01930-13) and to Conselho Nacional de Desenvolvimento Científico e Tecnológico - CNPq for the scholarship for scientific initiation awarded to the project.

\section{REFERENCES}

1. Zemlin WR. Princípios de Anatomia e Fisiologia em Fonoaudiologia. Porto Alegre: Artes Médicas Sul; 2000.

2. Yoshida M, Kikutani T, Tsuga K, Utanohara $\mathrm{Y}$, Hayashi R, Akagawa $Y$. Decreased tongue pressure reflects symptom of dysphagia. Dysphagia. 2006;21(1):61-5.

3. Dworkin JP, Aronson AE, Mulder DW. Tongue force in normals and in dysarthric patients with amyotrophic lateral sclerosis. J Speech and Hearing Research. 1980;23(4):828-37.

4. Proffit WR, Fields JR HW, Sarver DM. Ortodontia Contemporânea. Rio de Janeiro: Elsevier; 2007.

5. Furlan RMMM, Valentim AF, Motta AR, Barroso MFS, Costa CG, Las Casas EB. Quantitative methods for assessing tongue force. Rev. CEFAC. 2012;14(6):1215-25.

6. Perilo TVC, Motta AR, Las Casas EB, Saffar JME, Costa CG. Avaliação objetiva das forças axiais produzidas pela língua de crianças respiradoras orais. Rev Soc Bras Fonoaudiol. 2007;12(3):184-90.

7. Adams V, Mathisen B, Baines S, Lazarus C, Callister R. Reliability of measurements of tongue and hand strength and endurance using the lowa Oral Performance Instrument with healthy adults. Dysphagia. 2014;29(1):83-95.

8. Vitorino J. Effect of age on tongue strength and endurance scores of healthy Portuguese speakers. Int J Speech Lang Pathol. 2010;12(3):237-43.

9. Potter NL, Short R. Maximal tongue strength in typically developing children and adolescents. Dysphagia. 2009;24(4):391-7.

10. Steele CM, Bailey GL, Polacco REC, Hori SF, Molfenter SM, Oshalla $M$ et al. Outcomes of tongue-pressure strength and accuracy training for dysphagia following acquired brain injury. Int $\mathrm{J}$ Speech Lang Pathol. 2013;15(5):492-502.

11. Van Lierde KM, Bettens K, Luyten A, Plettinck $\mathrm{J}$, Bonte $\mathrm{K}$, Vermeersch $\mathrm{H}$ et al. Oral strength in subjects with a unilateral cleft lip and palate. Int $\mathrm{J}$ Pediatr Otorhinolaryngol. 2014;78(8):1306-10.

12. Felício CM, Dias FVS, Folha GA, Almeida LA, Souza JF, Anselmo-Lima WT et al. Orofacial motor functions in pediatric obstructive sleep apnea and implications for myofunctional therapy. Int J Pediatr Otorhinolaryngol. 2016;90(1):5-11.

13. Lazarus CL, Logemann JA, Pauloski BR, Rademaker AW, Helenowski IB, Vonesh EF et al. Effects of radiotherapy with or without chemotherapy on tongue strength and swallowing in patients with oral cancer. Head Neck. 2007;29(7):632-7.

14. Neel AT, Palmer PM, Sprouls G, Morrison L. Tongue strength and speech intelligibility in oculopharyngeal muscular dystrophy. J Med Speech Lang Pathol. 2006;14(4):273-7. 
15. Solomon NP. What is orofacial fatigue and how does it affect function for swallowing and speech? Semin Speech Lang. 2006;27(4):268-82.

16. Yeates EM, Molfenter SM, Steele CM. Improvements in tongue strength and pressure-generation precision following a tongue pressure training protocol in older individuals with dysphagia: three case reports. Clin Interv Aging. 2008;3(4):735-47.

17. Van Dyck C, Dekeyser A, Vantricht E, Manders E, Goeleven A, Fieuws S et al. The effect of orofacial myofunctional treatment in children with anterior open bite and tongue dysfunction: a pilot study. Eur J Orthod. 2016:38(3);227-34.

18. Kim HD, Choi JB, Yoo SJ, Chang MY, Lee SW, Park JS. Tongue-to-palate resistance training improves tongue strength and oropharyngeal swallowing function in subacute stroke survivors with dysphagia. J Oral Rehabil. 2017;44(1):59-64.

19. Robbins J, Kays SA, Gangnon RE, Hind JÁ, Hewitt $A L$, Gentry LR et al. The effects of lingual exercise in stroke patients with dysphagia. Arch Phys Med Rehabil. 2007;88(2):150-8.

20. Adams V, Mathisen B, Baines S, Lazarus C, Callister R. Reliability of measurements of tongue and hand strength and endurance using the lowa Oral Performance Instrument with elderly adults. Disabil Rehabil. 2015;37(5):389-95.

21. Watts CR. Measurement of hyolaryngeal muscle activation using surface electromyography for comparison of two rehabilitative dysphagia exercises. Arch Phys Med Rehabil. 2013;94(12):2542-8.

22. Yoshida M, Groher ME, Crary MA, Mann GC, Akagawa Y. Comparison of surface electromyographic (sEMG) activity of submental muscles between the head lift and tongue press exercises as a therapeutic exercise for pharyngeal dysphagia. Gerodontology. 2007;24(2):111-6.

23. Lenius K, Carnaby-Mann G, Crary EM. The relationship between lingual-palatal pressures and submental surface electromyographic signals. J Oral Rehabil. 2009;36(2):118-23.

24. Yoon WL, Khoo JKP, Liow SJR.Chin tuck against resistance (CTAR): new method for enhancing suprahyoid muscle activity using a shaker-type exercise. Dysphagia. 2014;29(2):243-8.

25. Furlan RMMM, Rezende BA, Motta AR. Comparison of the electric activity of the suprahyoid muscles during different lingual exercises. Audiol Commun Res. 2015;20(3):203-9.
26. Palmer PM, Luschei ES, Jaffe D, McCulloch TM. Contributions of individual muscles to the submental surface electromyogram during swallowing. J Speech Lang Hear Res. 1999;42(6):1378-91.

27. Marchesan IQ, Berretin-Félix G, Genaro KF. MBGR protocol of orofacial myofunctional evaluation with scores. Int J Orofacial Myol. 2012;38:38-77.

28. Rahal A, Lopasso FP. Eletromiografia dos músculos masseteres e supra- hióideos em mulheres com oclusão normal e com má oclusão classe I de Angle durante a fase oral da deglutição. Rev CEFAC. 2004;6(4):370-5.

29. De Luca CJ. The use of surface electromyography in biomechanics. J Appl Biomech. 1997;13:135-63.

30. Mukaka MM. A guide to appropriate use of correlation coeficiente in medical research. Malawi Med J. 2012;24(3):69-71.

31. Miller, JL, Watkin, KL, Chen, MF. Muscle, adipose, and connective tissue variations in intrinsic musculature of the adult human tongue. J Speech Lang Hear Res. 2002;45(1):51-65.

32. Gingrich LL, Stierwalt JAG, Hageman CF, LaPointe LL. Lingual propulsive pressures across consistencies generated by the anteromedian and posteromedian tongue by healthy young adults. J Speech Lang Hear Res. 2012;55(3):960.

33. Stal P, Marklund S, Thornell LE, De Paul R, Eriksson PO. Fibre composition of human intrinsic tongue muscles. Cells Tissues Organs. 2003;173(3):147-61. 